\title{
Capacidade de identificação de risco nutricional em idosos pelo Malnutrition Screening Tool em relação à miniavaliação nutricional.
}

\section{Capacity of identification of nutritional risk in elderly by Malnutrition Screening Tool in relation to mini nutritional assessment.}

\author{
Vanessa Teles Felinto' • Adriana Haack $^{2}$ - Alessandra Cedro da Silva Santos ${ }^{3}$
}

\begin{abstract}
RESUMO
Objetivo:Verificar a capacidade de identificação de risco nutricional pelo Malnutrition Screening Tool em comparação à Miniavaliação nutricional em idosos e identificar a prevalência de risco nutricional e desnutrição no público avaliado. Metodologia: Estudo transversal, analítico, com triagem e avaliação nutricional dos pacientes idosos (>=60 anos) hospitalizados, através dos métodos Malnutrition Screening Tool e Miniavaliação nutricional. Resultados: Foram avaliados 106 pacientes, com 48,1\% apresentando risco nutricional e 22,6\% apresentando desnutrição segundo a Miniavaliação nutricional. Pelo Malnutrition Screening Tool, encontrou-se risco nutricional em 52,8\% dos pacientes nas primeiras 48 horas de internação. Em relação à capacidade do Malnutrition Screening Tool em identificar risco nutricional nos pacientes idosos, em comparação com a Miniavaliação nutricional, observou-se associação entre os testes $(p<0,02)$, porém com tendência a um falso negativo, pois $19,8 \%$ dos pacientes classificados como sem risco nutricional pela Malnutrition Screening Tool apresentaram risco nutricional pela Miniavaliação nutricional. Conclusões: A utilização dos dois métodos se confirma para aqueles com risco nutricional, não podendo ser estendida para os sem risco nutricional pela divergência entre as análises, podendo ter o falso negativo nas avaliações da escala Malnutrition Screening Tool.
\end{abstract}

DESCRITORES: Idoso. Desnutrição.Triagem. Enfermagem Geriátrica.

\begin{abstract}
Objective: To verify the ability to identify nutritional risk by the Malnutrition Screening Tool compared to the Nutritional Mini-Assessment in the elderly and to identify the prevalence of nutritional risk and malnutrition in the evaluated public. Methodology: Cross-sectional, analytical study with screening and nutritional assessment of hospitalized elderly patients ( $>=60$ years), using the methods Malnutrition Screening Tool and Nutritional MiniAssessment. Results: A total of 106 patients were evaluated, with 48,1\% presenting nutritional risk and $22,6 \%$ presenting malnutrition according to the Mini Nutritional Assessment. Malnutrition Screening Tool found nutritional risk in 52,8\% of patients within the first 48 hours of hospitalization. Regarding the ability of Malnutrition Screening Tool in identifying nutritional risk in elderly patients, compared to the Nutritional Mini-Assessment, there was an association between the tests $(P<0,02)$, but with a tendency to a false negative, because $19,8 \%$ of patients classified as nonnutritional risk by the Malnutrition Screening Tool had nutritional risk by the Nutritional Mini-Assessment. Conclusions: The use of both methods is confirmed for those with nutritional risk, and cannot be extended to those without nutritional risk due to the divergence between the analyzes, and may have the false negative in the Malnutrition Screening Tool scale evaluations.
\end{abstract}

DESCRIPTORS: Elderly. Malnutrition. Screening. Geriatric Nursing. 
INTRODUÇÃO

A desnutrição é uma desordem comum no paciente idoso e está relacionada a importantes alterações clínicas e funcionais neste público, levando a efeitos adversos na composição corporal, maiores riscos para quedas, maior suscetibilidade a infecções, anemia, alterações cognitivas (delirium, depressão e etc), cicatrização deficiente, recuperação cirúrgica tardia, maior taxa de hospitalização e de readmissão, com consequente redução da qualidade de vida e aumento da mortalidade ${ }^{(1,2)}$.

Segundo o Inquérito Brasileiro de Avaliação Hospitalar (IBRANUTRI, 1998), a desnutrição estava presente em 48, I\% dos pacientes internados, sendo que $31,8 \%$ encontravam-se desnutridos nas primeiras 48 horas de internação e dentre os idosos a prevalência foi de $53 \%$. Também de acordo com o estudo BRAINS, em 2013, foi encontrado prevalência de déficit nutricional em $69,2 \%$ dos pacientes idosos nas primeiras 48 horas de internação hospitalar (38,4\% pacientes sob risco de desnutrição e 30,8\% desnutridos) ${ }^{(3,4,5)}$. Desde 1992, com a Conferência Internacional de Nutrição, a Organização Mundial de Saúde reconhece essa população como um público mais vulnerável nutricionalmente ${ }^{(6)}$.

Apesar dessa alta prevalência, a desnutrição frequentemente não é diagnosticada, favorecendo ao maior risco de complicações hospitalares, além de maior tempo de internação e aumento dos gastos ${ }^{(7)}$. Assim, a identificação precoce do risco de desnutrição através de ferramentas validadas e úteis desempenha um papel fundamental em termos de melhores desfechos clínicos $^{(5)}$.

A triagem nutricional consiste em um método rápido, que deve ser aplicado na admissão hospitalar e busca identificar indivíduos com risco para desnutrição, sinalizando precocemente ao profissional de saúde os pacientes que se beneficiariam de uma intervenção nutricional, favorecendo não só o paciente idoso, como o sistema de saúde como um todo $(6,8)$.

$\mathrm{Na}$ população idosa, a validade de um método de rastreio nutricional que exige aspectos da história do paciente pode ser afetada pela presença de delirium e demência (comum a esse público), subrelato de perda poderal, relatos de ingestão oral ou de outros fatores que não condizem com a realidade do paciente.Também, a utilização do Indice de Massa Corporal (IMC) e outras medidas antropométricas ou bioquímicas podem ser afetadas por mudanças de fluidos ou outras alterações fisiológicas, afetando assim o desempenho de ferramentas de rastreio validadas na prática clínica e levando a um subdiagnóstico da desnutrição. Por isso, é de extrema importância que o método escolhido seja validado para a população a que será aplicado. Dentre os vários métodos de rastreio nutricional validados para a prática clínica existem a Mini Avaliação Nutricional (MAN) e o Malnutrition Screening Tool (MST) $)^{(9,10)}$.

A MAN é um método validado para idosos, consiste em questionário dividido em cinco partes, sendo a primeira de triagem e outras quatro partes relacionadas à avaliação antropométrica, questionário alimentar, avaliação global e auto avaliação do idoso. Cada item gera um score e, de acordo com a pontuação obtida, se classifica em bem nutrido, risco para desnutrição e desnutrido(5,11).

O MST é um método validado para a população adulta que busca identificar risco de desnutrição em pacientes durante a admissão hospitalar. É composto por apenas 3 questões relacionadas a perda de peso e ao apetite ${ }^{(12)}$.
Nesse sentido, o objetivo desse estudo foi verificar a capacidade de identificação de risco nutricional pelo MST em comparação à MAN na população idosa (público em que foi realizada a pesquisa) além de identificar a prevalência de risco nutricional e desnutrição no público avaliado.

\section{MÉTODO}

Estudo transversal e analítico, realizado pela própria pesquisadora, no Pronto Socorro de um hospital público do Distrito Federal. Para a amostragem, foram confirmadas no Núcleo de Internação e Alta da Unidade Hospitalar, as informações disponíveis sobre internação de idosos $(\geq 60$ anos) e, a partir daí, estabeleceu-se uma coleta de informações por 3 meses - setembro a novembro de 2016, de todos idosos (as) internados (as) no pronto socorro com características relevantes (critérios de inclusão) para o estudo. Dessa forma, foi utilizado o método de amostragem não casual (não probabilística) e os casos estudados faziam parte do atendimento estabelecido no serviço estando de acordo com o objetivo da pesquisa de testar os instrumentos na amostra construída.

Foram incluídos todos os idosos, de ambos os sexos, admitidos no período determinado, na unidade de emergência e avaliados nas primeiras 48 horas de internação.

Foram excluídos os pacientes incapazes de informar os dados necessários para a realização das avaliações, que não tinham acompanhantes aptos para esse fim, que não desejassem participar do estudo e pacientes com diagnóstico de morte encefálica. Todos os pacientes tiveram participação voluntária após detalhamento dos objetivos do estudo e da forma de participação, com assinatura do Termo de Consentimento Livre e Esclarecido - TCLE pelo próprio paciente ou acompanhante.

A pesquisadora foi devidamente treinada para a coleta dos dados, o uso dos dois instrumentos e o modelo de pesquisa, assim como, as responsabilidades dos pesquisadores foram avaliados e aprovados pelo Comitê de Ética em Pesquisa da Fundação de Ensino e Pesquisa em Ciências da Saúde, em 01/07/2016 -Parecer Consubstanciado $n^{\circ} 1.621 .570$, estando adequados para a segurança na coleta de dados e seguiram as normas da Resolução $n^{\circ} 466 / 2012$ do CNS que minimizam os riscos para os participantes.

Todo paciente que assinou o TCLE teve o seu estado nutricional triado, no prazo de 48 horas após sua internação no Pronto Socorro do HRC, pela MST e pela MAN.

A MAN consiste em um questionário composto por 18 questões agrupadas em 3 partes: antropometria (faixa de IMC, perda de peso, circunferência do braço e da panturrilha), avaliação clínica (quantidade de medicamentos em uso, mobilidade, estilo de vida, tipo de residência, presença de úlceras em pele, estresse psicológico ou problemas neuropsicológicos), avaliação dietética (autonomia na alimentação, qualidade e número de refeições, auto-percepção sobre saúde e nutrição). $\mathrm{Na}$ sua aplicação, foi realizada a parte da triagem (primeiras 6 questões) na qual classifica o paciente como sem risco (score igual ou maior que 12), tornando desnecessária o restante da avaliação, ou sob risco de desnutrição (score igual ou inferior a II), indicando a necessidade de aplicação da parte de avaliação global (questões restantes) para obter o escore do estado nutricional. O somatório da triagem com o da avaliação global permitiu a determinação da presença de Risco Nutricional ou de Desnutrição, de acordo com o escore obtido (pontuação $<17$ = Desnutrição; de 17 a 23,5 = Risco de Desnutrição; $\geq 24$ 
$=$ Sem Risco Nutricional) ${ }^{(5)}$.

método de triagem nutricional MST é composto por 3 questões relacionadas à redução da ingestão alimentar, perda de peso não intencional e quantidade da perda ponderal. Resulta em pontuação de zero a 13, de forma que, ao ser aplicado, se $\circ$ paciente receber score $\geq 2$, será classificado Com Risco Nutricional e abaixo desse score, Sem Risco Nutricional( ${ }^{(12)}$.

A análise estatística foi realizada por meio do software IBM SPSS 22, utilizando o índice de confiabilidade de 95\%. Com o objetivo de verificar o risco nutricional dos pacientes em relação à MAN e à MST, foi verificado se havia associação nas distribuições dos pacientes em relação à classificação, por meio do teste do Qui quadrado. Também foi realizado a análise de concordância de Kappa. Para realização da análise considerouse que todos os pacientes desnutridos na MAN estavam em risco de desnutrição, para dessa forma, verificar a possibilidade de avaliar se a MST traz uma classificação similar.

\section{RESULTADOS}

A amostra foi composta por 106 pacientes, divididos em $58(54,7 \%)$ do sexo masculino e $48(45,3 \%)$ do sexo feminino. A média de idade foi de 73,26 $\pm 8,3$ anos, com distribuição normal, segundo teste de Kolmogorov-Smirnov ( $p>0,05)$. Não houve diferença significativa entre os dados em relação à idade e sexo $(p>0,05)$, podendo ser tratado o grupo como único, independente do sexo (Gráfico I).

Gráfico I. Distribuição da idade, segundo faixa etária, dos idosos internados no Pronto Socorro de um hospital público do Distrito Federal, de setembro a novembro de $2016(n=106)$.

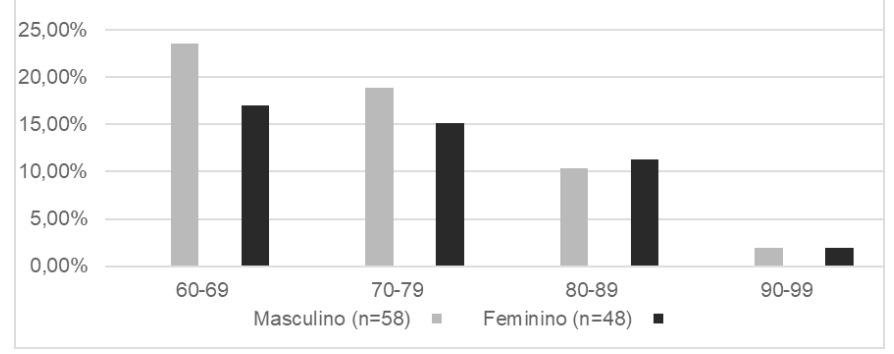

Fonte: Própria da pesquisa. da cidade onde se localizou o estudo possui renda baixa, situação que impacta diretamente no estado nutricional da população, em especial dos idosos ${ }^{(13)}$.

Nas análises descritivas dos grupos em relação ao Risco Nutricional, verificou-se um percentual alto de pacientes Com Risco (48, I\%) e Desnutridos (22,6\%) segundo a MAN.Também segundo a MST, esse percentual foi elevado, encontrando-se que $52,8 \%$ dos pacientes estavam Com Risco Nutricional nas primeiras 48 horas de internação (Tabela I).

Tabela I: Estado nutricional dos idosos internados no Pronto Socorro do Hospital público do Distrito Federal de setembro a novembro de 2016 .

\begin{tabular}{|l|c|c|l|c|c|}
\hline \multicolumn{3}{|c|}{ DIAGNOSTICO MAN $^{\text {a }}$} & \multicolumn{3}{c|}{ DIAGNOSTICO MST $^{\text {b }}$} \\
\hline & $\mathrm{N}$ & $\%$ & & $\mathrm{~N}$ & $\%$ \\
\hline Sem Risco nutricional & 31 & 29,2 & Sem Risco Nutricional & 50 & 47,2 \\
\hline $\begin{array}{l}\text { Com Risco } \\
\text { nutricional }\end{array}$ & 51 & 48,1 & $\begin{array}{l}\text { Com Risco } \\
\text { Nutricional }\end{array}$ & 56 & 52,8 \\
\hline
\end{tabular}

\begin{tabular}{|l|c|c|l|c|c|}
\hline Desnutrido & 24 & 22,6 & - & -- & -- \\
\hline Total & 106 & 100 & Total & 106 & 100 \\
\hline & a MAN = Mini Avaliação Nutricional $;{ }^{\text {b }}$ MST = Malnutrition Screening Tool \\
Fonte: Própria da pesquisa.
\end{tabular}

A MAN possibilita rastrear o estado nutricional dos pacientes como Sem Risco, Com risco e Desnutrido e a MST só classifica como Sem ou Com Risco Nutricional, dessa forma, para a análise, todos os pacientes desnutridos na MAN foram incluídos no grupo dos pacientes classificados como com Risco Nutricional, para verificar a possibilidade de avaliar se a MST traz uma classificação similar (Tabela 2).

Tabela 2: Comparação dos diagnósticos encontrados pela Malnutrition Screening Tool (MST) e pela Mini Avaliação Nutricional (MAN) em idosos internados no Pronto Socorro de um hospital público do Distrito Federal, de setembro à novembro de 2016.

\begin{tabular}{|c|c|c|c|c|}
\hline & & \multicolumn{2}{|c|}{ DIAGNOSTICO MAN } & \multirow{3}{*}{$\begin{array}{c}\text { Total } n \\
(\%)\end{array}$} \\
\hline & & $\begin{array}{l}\text { Sem risco } \\
\text { nutricional }\end{array}$ & $\begin{array}{l}\text { Com risco } \\
\text { nutricional }\end{array}$ & \\
\hline & & $\mathrm{n}(\%)$ & $\mathrm{n}(\%)$ & \\
\hline \multirow{4}{*}{$\begin{array}{l}\text { DIAGNOSTI- } \\
\text { CO MST }\end{array}$} & $\begin{array}{l}\text { Sem risco } \\
\text { nutricional }\end{array}$ & $29(27)$ & $21(20)$ & $50(47)$ \\
\hline & $\%(n)$ & & & \\
\hline & $\begin{array}{l}\text { Com risco } \\
\text { nutricional }\end{array}$ & $2(2)$ & $54(5 I)$ & $56(53)$ \\
\hline & $\%(n)$ & & & \\
\hline Total & & $31(29)$ & $75(7 \mathrm{I})$ & $106(100)$ \\
\hline
\end{tabular}

Fonte: Própria da pesquisa.

Observa-se que há associação entre os testes, com um $\mathrm{p}<0,02$. Porém há uma tendência a um falso negativo, pois $20 \%$ do total de pacientes que foram classificados como Sem Risco Nutricional pela MST, apresentaram Risco Nutricional pela MAN.

Já em relação à presença de Risco Nutricional, os dois métodos tendem a ter uma classificação similar, como observado pelos 54 pacientes que foram classificados como Com Risco Nutricional por ambos os métodos, representando $50,9 \%$ da população total.

Quando avaliado a amostra por meio da concordância de Kappa, foi verificado o índice de 0,56, o que representa uma concordância moderada entre as avaliações, com $\mathrm{p}<0,0 \mathrm{l}$ e intervalo de confiança de $95 \%$. A concordância foi usada para os dois conjuntos de dados de maneira complementar ao teste Qui quadrado e a intensidade da concordância foi ser interpretada levando-se em consideração a análise conjunta e a similaridade do perfil dos idosos na emergência, portanto, analisado de maneira cuidadosa na determinação de qual método seria melhor que o outro na identificação de risco nutricional.

\section{DISCUSSÃO}

Como mostrado em alguns estudos, a prevalência de desnutrição protéico-energética em idosos hospitalizados é alta e se associa diretamente à piores desfechos clínicos, maior morbidade e mortalidade. Apesar disso, estudos também revelam que apenas uma minoria dos pacientes idosos hospitalizados que se encontra desnutrida ou com algum risco para desnutrição recebe suporte nutricional adequado, confirmando a necessidade de intervenções nutricionais 
precoces nesse grupo(7).

Ao longo das últimas décadas, vários métodos de triagem e avaliação nutricional foram desenvolvidos com o objetivo de facilitar o rastreio do estado nutricional dos pacientes, de forma a colaborar com a equipe clínica a tratar os problemas nutricionais precocemente. A fim de avaliar qual ferramenta é melhor para determinada população é importante a realização de estudos comparando várias ferramentas nesse mesmo público ${ }^{(5)}$. Diante disso, pela necessidade do serviço da otimização do tempo dedicado à realização da triagem nutricional, buscou-se comparar nesse trabalho a eficácia do método MST em relação aos resultados obtidos pela MAN, na triagem dos pacientes idosos internados em um pronto socorro de hospital público do DF. O primeiro é um método rápido e de fácil aplicação e o segundo é método de avaliação de risco nutricional nutricional e desnutrição específico para esse público.

Os resultados desse trabalho demonstraram uma alta prevalência de Risco Nutricional (48,1\%) e de Desnutrição $(22,6 \%)$, segundo a MAN, nos pacientes idosos internados durante o período de coleta. Também encontramos uma alta prevalência de Risco Nutricional segundo à MST (52,8\%), porém como exposto anteriormente, esse valor pode estar mascarado pela tendência a um falso negativo dos pacientes classificados como Sem Risco Nutricional por esse método, podendo ser o número de pacientes em risco ainda maior. Esses dados convergem com um estudo realizado com 86 idosos, triados nas primeiras 72 horas de internação, em que o risco nutricional esteve presente em $53,5 \%$ dessa população e em outro estudo com 90 idosos, em que a prevalência de risco nutricional foi de $46,7 \%(14,15)$.

Os resultados obtidos mostraram que a aplicação do MST é segura para aqueles pacientes que possuem risco nutricional, quando esse resultado é comparado ao obtido pela MAN. porém, o mesmo raciocínio não pode ser estendido aos pacientes que se apresentam Sem Risco Nutricional, segundo - MST, pela possibilidade de um falso negativo em relação aos resultados obtidos pela MAN. Assim, a utilização dos dois métodos de triagem possui uma característica de confirmação para aqueles que possuem Risco Nutricional, não podendo ser estendida para os sem risco nutricional, pela divergência entre as análises, podendo ter o falso negativo nas avaliações da escala MST. Uma explicação para isso pode ser a falta no MST de questões que avaliem itens importantes no contexto do paciente idoso.

Um item importante no contexto dessa população diz respeito a presença de algum transtorno cognitivo ou quadro de depressão. Achados de Maseda et al( $^{(8)}$ demonstram associação direta de desnutrição em pacientes idosos com depressão, além de citar outros estudos que encontraram resultados semelhantes. A demência também é preditor independente para risco de desnutrição, quando comparado a pacientes com nível cognitivo normal(16).

Um aspecto também importante é a avaliação de fragilidade do idoso, que como demonstrado por Maseda et al(8) tem forte associação com a presença de desnutrição ou de risco nutricional, porém também não é avaliado pelo MST.

Outro item que não é considerado no MST e que tem grande importância no processo do envelhecimento é o que se relaciona à capacidade funcional do idoso. Isso determina a sua capacidade de realizar tarefas básicas de autocuidado e viver de forma independente, incluindo a mobilidade, a independência física e financeira e a capacidade de se alimentar, que contempla desde a aquisição até a efetiva absorção do alimento. Todos esses itens demonstram ter associação significativa com a ingestão alimentar e o estado nutricional(14).

Uso de medicamentos também tem influência no estado nutricional, já que promove alteração de paladar, anorexia, além de má absorção, de forma que estudos já mostram a associação de polifarmácia (uso de cinco ou mais medicamentos por dia) com piores scores nutricionais na $\mathrm{MAN}^{(8)}$.

Em relação aos indicadores sócio econômicos, a população estudada tem renda baixa, situação que apresenta associação com o estado nutricional, como demonstrado por Jang et $\mathrm{al}^{(16)}$, que em seu estudo encontraram que dificuldades financeiras e baixa renda se associam com risco nutricional e desnutrição na população idosa. Pereira, Spyrides e Andrade (13) também encontraram associa $\neg$ ção positiva do IMC com a renda mensal per ca $\urcorner$ pita de idosos.

O principal objetivo de se realizar rastreamento nutricional de pacientes internados, em especial dos idosos, consiste em identificar pacientes Com Risco Nutricional ou com um quadro de Desnutrição já estabelecido para que, assim, seja iniciado um plano nutricional adequado e, caso se faça necessário, com o uso de suplementos nutricionais.

Já está estabelecido que a triagem, seguida da avaliação nutricional do paciente, identifica precocemente alterações no estado nutricional, levando à uma melhor assistência, de forma que o paciente evolua com correção desse quadro e com redução das complicações associadas a ele $^{(1)}$. Nesse contexto, o MST se mostrou um método de triagem efetivo para rastreio nutricional nos pacientes idosos que participaram dessa pesquisa quando comparado à MAN e por ser uma ferramenta rápida, de fácil utilização e sensível para detectar Risco Nutricional em idosos, recomendamos o seu uso também para esse público em nível hospitalar.

Em contrapartida, deve-se dar também atenção aos pacientes que forem classificados como sem risco por esse método, para que outras características que não são levadas em consideração e que são importantes no contexto do paciente idoso, façam parte desse rastreio, tornando o resultado da triagem nutricional mais fidedigno.

É de grande importância para os serviços de saúde a realização de trabalhos que procuram avaliar o estado nutricional da população atendida e propor adequações e melhorias à equipe de trabalho. Todo esse esforço é importante para o alcance de uma maior capacitação profissional e, consequentemente, melhor assistência ao paciente internado, mesmo em um ambiente de pronto socorro, onde a dinâmica do trabalho frequentemente limita a uma atenção mais individualizada por parte da equipe à pessoa que está sendo cuidada.

\section{CONCLUSÃO}

O método de triagem nutricional MST mostrou ter relação com a MAN quando os pacientes são classificados como Com Risco Nutricional pelo primeiro. Contudo, boa parte dos que foram classificados como Sem Risco Nutricional pelo MST apresentavam-se Com Risco Nutricional ou Desnutridos pela MAN, demonstrando um resultado falso negativo. Isso caracteriza o MST como um método rápido, de fácil utilização e capaz de identificar os pacientes idosos em risco nutricional. No entanto, há uma limitação para seu uso, já que, para um número considerável de indivíduos, não seria dada a devida 
intervenção nutricional que deve ser direcionada aos pacientes com risco nutricional.

Na população estudada, encontrou-se uma alta prevalência de Risco Nutricional (48, I\%) e de Desnutrição (22,6\%) segundo a MAN e também segundo à MST (52,8\%), demonstrando a necessidade do rastreio nutricional nesse público na busca de melhores desfechos durante a internação hospitalar desses pacientes.

\section{REFERÊNCIAS}

1. Harding KM, Dyo M, Goebel JR, Gorman N, Levine $J$. Early malnutrition screening and low cost protein supplementation in elderly patients admitted to a skilled nursing facility. Appl Nurs Res. I de agosto de 2016;31:29-33.

2. Guyonnet S, Rolland Y. Screening for Malnutrition in Older People. Clin Geriatr Med. Agosto de 20I5; 3I(3):429-37. doi: 10.1016/j.cger.2015.04.009. Epub 2015 May 13.

3. Borghi R, Meale MMS, França JID, Pereira MAG, Damião AOMC. Eficácia da intervenção nutricional em pacientes hospitalizados com desnutrição: subanálise do estudo BRAINS. Rev Bras Nutr Clin 2015; 30 (I):38.

4. Hordonho AAC, Santos DC, Powell JRF. Perfil antropométrico e força muscular em adultos e idosos de um hospital público de referência em doenças infectocontagiosas em Maceió-AL (dados preliminares). Braz J Hea Rev, Curitiba, v. 2, n. 2, p. 6, I 245-I 274, mar./ apr. 2019.

5. Rosa EPC, Bacalhau SPOS, Silva AS, Santos IAM, Borges FDS, Silva GA et al. Risco e evolução do estado nutricional de adultos e idosos hospitalizados com distúrbios neurológicos. Nutr clín diet hosp. 2019; 39(2):46-53.

6. Baek MH, Heo YR. Evaluation of the efficacy of nutritional screening tools to predict malnutrition in the elderly at a geriatric care hospital. Nutr Res Pract. 2015;9(6):637-43.

7. Zhou J, Wang M,Wang H, Chi Q. Comparison of two nutrition assessment tools in surgical elderly inpatients in Northern China. Nutr J. 20I5; I4:68.

8. Maseda A, Gómez-Caamaño S, Lorenzo-López L, López-López R, Diego-Diez C, Sanluís-Martínez V et al. Health determinants of nutritional status in community-dwelling older population: the VERISAÚDE study. Public Health Nutrition. 2016; 19(12):2220-8.
9. Marshall S, Young A, Bauer J, Isenring E. Malnutrition in Geriatric Rehabilitation: Prevalence, Patient Outcomes, and Criterion Validity of the Scored Patient-Generated Subjective Global Assessment and the Mini Nutritional Assessment. J Acad Nutr. Diet 2016; I I6(5): 785-94.

10. Marshall S, Young A, Bauer J, Isenring E Nutrition Screening in Geriatric Rehabilitation: Criterion (Concurrent and Predictive) Validity of the Malnutrition Screening Tool and the Mini Nutritional Assessment_ Short Form. J Acad Nutr Diet. 20 I6; I I 6(5): 795-80I.

11. Pardal LP, Montells LP, Álvarez LR. Mayores que viven solos y malnutrición. Estudio SOLGER. Aten Primaria.2017;49(8):450-458.

12. VanDerBosch G, Sulo S, Dziadosz M, Kozmic SE, Sorkin K, Lanctin D et al. Similar health economic outcomes in low-risk and high-risk malnourished inpatients as screened by the Malnutrition Screening Tool after delivery of oral nutritional supplements. Nutrition. 2019; 67-68.

13. Pereira IFS, Spyrides MHC, Andrade LMB. Estado nutricional de idosos no Brasil: uma abordagem multinível. Cad Saúde Pública. 2016; 32(5).

14. Santos CA, Ribeiro AQ, Rosa COB, Ribeiro RCL. Depressão, déficit cognitivo e fatores associados à desnutrição em idosos com câncer. Ciênc Saúde Coletiva. 2015; 20 (3).

15. Arruda NR, Oliveira ACCC, Garcia, LJC. Risco nutricional em idosos: comparação de métodos de triagem nutricional em hospital público. RASBRAN. 2019; 10 (I):59-65.

16. Jang JW, Kim Y, Choi YH, Lee JM, Yoon B, Park KW et al. Association of Nutritional Status with Cognitive Stage in the Elderly Korean Population: The Korean Brain Aging Study for the Early Diagnosis and Prediction of Alzheimer's Disease. J Clin Neurol. 2019 Jul; I 5(3):292300. 